\title{
Identification of RegIV as a Novel GLI1 Target Gene in Human Pancreatic Cancer
}

\author{
Feng Wang', Ling $\mathrm{Xu}^{2}$, Chuanyong Guo ${ }^{2}$, Aiwu $\mathrm{Ke}^{2}$, Guoyong $\mathrm{Hu}^{2}$, Xuanfu $\mathrm{Xu}^{2}$, Wenhui Mo ${ }^{2}$, Lijuan \\ Yang ${ }^{1}$, Yinshi Huang ${ }^{2}$, Shanshan $\mathrm{He}^{2}$, Xingpeng Wang ${ }^{1,2 *}$
}

1 Department of Gastroenterology, The First People's Hospital, Shanghai Jiao Tong University School of Medicine, Shanghai, People's Republic of China, 2 Department of Gastroenterology, The Tenth People's Hospital of Shanghai, Tongji University, Shanghai, People's Republic of China

\begin{abstract}
Background and Aims: GLI1 is the key transcriptional factor in the Hedgehog signaling pathway in pancreatic cancer. RegIV is associated with regeneration, and cell growth, survival, adhesion and resistance to apoptosis. We aimed to study RegIV expression in pancreatic cancer and its relationship to GLI1.

Methods: GLI1 and RegIV expression were evaluated in tumor tissue and adjacent normal tissues of pancreatic cancer patients and 5 pancreatic cancer cell lines by qRT-PCR, Western blot, and immunohistochemistry (IHC), and the correlation between them. The GLI1-shRNA lentiviral vector was constructed and transfected into PANC-1, and lentiviral vector containing the GLI1 expression sequence was constructed and transfected into BxPC-3. GLI1 and RegIV expression were evaluated by qRT-PCR and Western blot. Finally we demonstrated RegIV to be the target of GLI1 by chromatin immunoprecipitation (CHIP) and electrophoretic mobility shift assays (EMSA).

Results: The results of IHC and qRT-PCR showed that RegIV and GLI1 expression was higher in pancreatic cancer tissues versus adjacent normal tissues $(p<0.001)$. RegIV expression correlated with GLI1 expression in these tissues $(R=0.795$ $p<0.0001)$. These results were verified for protein $(R=0.939, p=0.018)$ and mRNA expression $(R=0.959, p=0.011)$ in 5 pancreatic cancer cell lines. RegIV mRNA and protein expression was decreased $(94.7 \pm 0.3 \%, 84.1 \pm 0.5 \%$; respectively) when GLI1 was knocked down $(82.1 \pm 3.2 \%, 76.7 \pm 2.2 \%$; respectively) by the RNAi technique. GLI1 overexpression in mRNA and protein level $(924.5 \pm 5.3 \%, 362.1 \pm 3.5 \%$; respectively) induced RegIV overexpression $(729.1 \pm 4.3 \%, 339.0 \pm 3.7 \%$; respectively). Moreover, CHIP and EMSA assays showed GLI1 protein bound to RegIV promotor regions (GATCATCCA) in pancreatic cancer cells.
\end{abstract}

Conclusion: GLI1 promotes RegIV transcription by binding to the RegIV gene promoter in pancreatic cancer.

Citation: Wang F, Xu L, Guo C, Ke A, Hu G, et al. (2011) Identification of RegIV as a Novel GLI1 Target Gene in Human Pancreatic Cancer. PLoS ONE 6(4): e18434. doi:10.1371/journal.pone.0018434

Editor: Vladimir N. Uversky, University of South Florida College of Medicine, United States of America

Received October 10, 2010; Accepted March 4, 2011; Published April 11, 2011

Copyright: (C) 2011 Wang et al. This is an open-access article distributed under the terms of the Creative Commons Attribution License, which permits unrestricted use, distribution, and reproduction in any medium, provided the original author and source are credited.

Funding: This work was supported in part by National Natural Science Foundation of China (No. 81072065), Foundation for Shanghai Science and Technology Committee (No. 09JC1412200, No. 09410705400), and Doctoral Fund of Ministry of Education of China (No. 20090072110022). The funders had no role in study design, data collection and analysis, decision to publish, or preparation of the manuscript.

Competing Interests: The authors have declared that no competing interests exist.

* E-mail: wangxingpeng@hotmail.com

\section{Introduction}

Pancreatic cancer $(\mathrm{PG})$ is the fourth most common cancerrelated cause of mortality in the Western world [1-3] and has a dismal prognosis despite considerable progress in management. The median survival of PC is less than 6 months; the 5-year survival rate is less than $5 \%[1,2]$. More than $80 \%$ present with unresectable disease; one-third have local disease while the remainder have distant metastases. Research over the last two decades has shown that PG is a genetic disease fundamentally, caused by inherited germline and acquired somatic mutations in cancer-associated genes. Tumor progression model for PG in which the pancreatic ductal epithelium progresses from normal to increased grades of pancreatic intraepithelial neoplasia (PanINs) to invasive cancer. Multiple alterations in genes that are important in PC progression have been identified, for example K-ras, INK4A, p53, and SMAD4/DPC4 [4,5]. PG is characterized by near- universal mutations in K-ras and frequent deregulation of crucial embryonic signalling pathways, including the Hedgehog $(\mathrm{HH})$ signaling pathway $[6,7]$. A better understanding of the mechanisms underlying the development of PC might help to improve early diagnosis and potentially identify molecular therapeutic targets.

The hedgehog $(\mathrm{HH})$ signaling pathway was first identified in the embryonic development of Drosophila [8] and has been shown to be crucial for growth and patterning in the pancreas during embryonic development. HH signaling regulates cell differentiation and organ formation during embryonic development, and is expressed in pancreatic epithelial cells $[9,10]$. Constitutive activation of $\mathrm{HH}$ signaling is detected in a variety of human cancers, including pancreatic cancer [9-13]. Given its misexpression in both metastatic pancreatic cancer cell lines and in precursor lesions (PanIN) [14], HH signal activation may be involved in both early and late pancreatic tumorigenesis. 
Of the three mammalian ligands in the $\mathrm{HH}$ family, Sonic $(\mathrm{SHH})$, Desert $(\mathrm{DHH})$, and Indian (IHH) Hedgehog [15], the former has been associated with both pancreatic organogenesis and pancreatic cancer. $\mathrm{HH}$ signals are transmitted and modified by two transmembrane proteins, patched (PTCH) and smoothened (SMO), and by downstream transcription factors that are members of the glioma-associated oncogene (GLI) family (GLI1, 2, and 3). GLI2 and GLI3 have transactivation and repressive domains, whereas GLI1 likely functions only as a transactivator and transcriptional target of the $\mathrm{HH}$ pathway itself [16-19]. The regenerating gene (Reg) family, a group of small secretory proteins, is involved in cell proliferation or differentiation in digestive organs [20], is upregulated in several gastrointestinal cancers, and functions as trophic or antiapoptotic factors [21-23]. RegIV, a member of the regenerating gene family, is involved in digestive tract malignancies, including the stomach [24], colorectum $[25,26]$, and pancreas $[27,28]$, as well as in benign diseases such as ulcerative colitis [29]. RegIV overexpression in tumor cells has been associated with cell growth, survival, adhesion, and resistance to apoptosis. Recently, RegIV overexpression was reported to be associated with the initiation and progression of pancreatic cancer, and was suggested as a promising tumor marker to screen early stage PC and target for adjuvant therapy in PC $[28,30]$.

The functions of GLIl and RegIV appeared to be similar in our review of the literature; thus, we investigated the expression and correlation of GLIl and RegIV in PC tissues and cell lines. We also explored the possible mechanism between GLIl and RegIV, by using ChIP and EMSA assays.

\section{Materials and Methods}

\section{Cell lines and tissues}

Human pancreatic cancer cell lines, PANC-1, AsPC-1, BxPG3, CaPan-2 and SW1990, were purchased from Chinese Academy of Sciences Committee Type Culture Collection cell bank. PANC-1 was cultured in Dulbecco's Modified Eagle's Medium (DMEM) (Gibco BRL, USA), the other types of Cells were cultured in RPMI-1640 (Gibco BRL, USA), and all the mediums were supplemented with 10\% FBS (Gibco BRL, USA), penicillin $\mathrm{G}(100 \mathrm{U} / \mathrm{ml})$, streptomycin $(100 \mathrm{ug} / \mathrm{ml})$. Cells were incubated at $37^{\circ} \mathrm{C}$ with $5 \% \mathrm{CO} 2$. Twelve pairs of $\mathrm{PG}$ and corresponding non-cancerous pancreas tissues were obtained from Shanghai Tenth People's Hospital with full written ethical consent. None of these patients had received chemotherapy or radiation therapy prior to cancer resection. Another 9 paired tissues slices was obtained from pathology department of
Shanghai Tenth People's Hospital. The study was approved by the Ethical Committee of Tongji University School of Medicine and Life Sciences.

Short Hairpin RNA (shRNA) Design and Vector Production

Interfering sequences corresponding to distinct regions of GLIlmRNA, as well as negative control with no homology for human or mouse genes were designed by Shanghai GeneChem Biotech (Table 1). Three siRNA duplexes were screened for GLIl knock-down by Western blot analysis in cotransfection experiments with GLI1 expression plasmid in HEK 293T cells. The most successful sequence and one non-silencing Luciferase sequence were designed into a shRNA oligonucleotide template consisting of sense, hairpin loop, antisense, and terminator sequences, all of which were flanked by restriction enzyme sites to facilitate directional sub-cloning. The resulting vectors encoded GFP under transcriptional control of the EF1 promoter and a $\mathrm{H} 1$ promoter upstream of cloning restriction sites $(M l u \mathrm{I}$ and $C l a \mathrm{I})$ to allow the introduction of oligonucleotides encoding shRNAs. Either shRNA against GLI1 or a nonsilencing-Luciferase shRNA was located under the $\mathrm{H} 1$ promoter (Figure 1). The correct insertion of the specific shRNA was further confirmed by direct DNA sequencing.

For production of the lentiviral vector, HEK 293T cells were cultured to $30-40 \%$ confluence by the following day. The next day, the medium was replaced with DMEM/10\% FBS without antibiotics. Subsequently, $20 \mu \mathrm{g}$ of shRNA plasmid DNA (nonsense shRNA or GLIl targeting shRNA; GeneChem Biotech, Shanghai, China), $7.5 \mu \mathrm{g}$ pMD2G, $10 \mu \mathrm{g}$ pRsv-Rev, and $15 \mu \mathrm{g}$ pMDLg-pRRE were mixed with sterile $\mathrm{dd}_{2} \mathrm{O}$ to a final volume of $1800 \mu \mathrm{l}$, then mixed with $200 \mu \mathrm{l}$ of $2.5 \mathrm{M} \mathrm{CaCl}_{2}$. The DNA mix was oxygenated and $2000 \mu \mathrm{l} 2 \times \mathrm{PBS}(\mathrm{pH} 7.05)$ added in drops, and incubated at room temperature for 30 minutes. The transfection mixture was added to its respective plates and incubated overnight. The medium was replaced after 12 hours with DMEM supplemented with 10\% FBS. After 48 hours, the conditioned medium containing shRNA lentivirus was collected and filtered through $0.45-\mu \mathrm{m}$ pore size cellulose acetate filters, and stored on ice. The virus was concentrated by spinning at 70,000 G for 2 hours and resuspended with $500 \mu \mathrm{l}$ PBS. The transduction unit (TU) titer was assessed on HEK 293T cells in the presence of polybrene $8 \mu \mathrm{g} / \mathrm{mL}$ (Sigma-Aldrich, St. Louis, MO, USA). Titers of $2-5 \times 10^{8} \mathrm{TU} / \mathrm{ml}$ were routinely achieved.

\section{Overexpression-GLI1 Lentiviral Vector Construction}

Human GLIl cDNA was purchased from Open-Biosystem (USA). The complete cDNA sequence of GLIl was generated by

Table 1. Sequences of primers used in this study for GLI1-shRNA constructs.

\begin{tabular}{|c|c|c|c|c|}
\hline $5^{\prime}$ & & STEMP & Loop & STEMP \\
\hline \multirow[t]{2}{*}{ shGLI1-1 } & CGCGTCCCC & CTCCACAGGCATACAGGAT & TTCAAGAGA & ATCCTGTATGCCTGTGGAG \\
\hline & CGATTTCCAAAAA & CTCCACAGGCATACAGGAT & TCTCTTGAA & ATCCTGTATGCCTGTGGAG \\
\hline \multirow[t]{2}{*}{ shGLI1-2 } & CGCGTCCCC & CGTGAGCCTGAATCTGTGTAT & TTCAAGAGA & ATACACAGATTCAGGCTCACG \\
\hline & CGATTTCCAAAAA & CGTGAGCCTGAATCTGTGTAT & TCTCTTGAA & ATACACAGATTCAGGCTCACG \\
\hline \multirow[t]{2}{*}{ shGLI1-3 } & CGCGTCCCC & GCTCAGCTTGTGTGTAATTAT & TTCAAGAGA & ATAATTACACACAAGCTGAGC \\
\hline & CGATTTCCAAAAA & GCTCAGCTTGTGTGTAATTAT & TCTCTTGAA & ATAATTACACACAAGCTGAGC \\
\hline \multirow[t]{2}{*}{ scramble } & CGCGTCCCC & GCCAGCGTTAACCAGACTA & TTCAAGAGA & TAGTCTGGTTAACGCTGGC \\
\hline & CGATTTCCAAAAA & GCCAGCGTTAACCAGACTA & TCTCTTGAA & TAGTCTGGTTAACGCTGGC \\
\hline
\end{tabular}




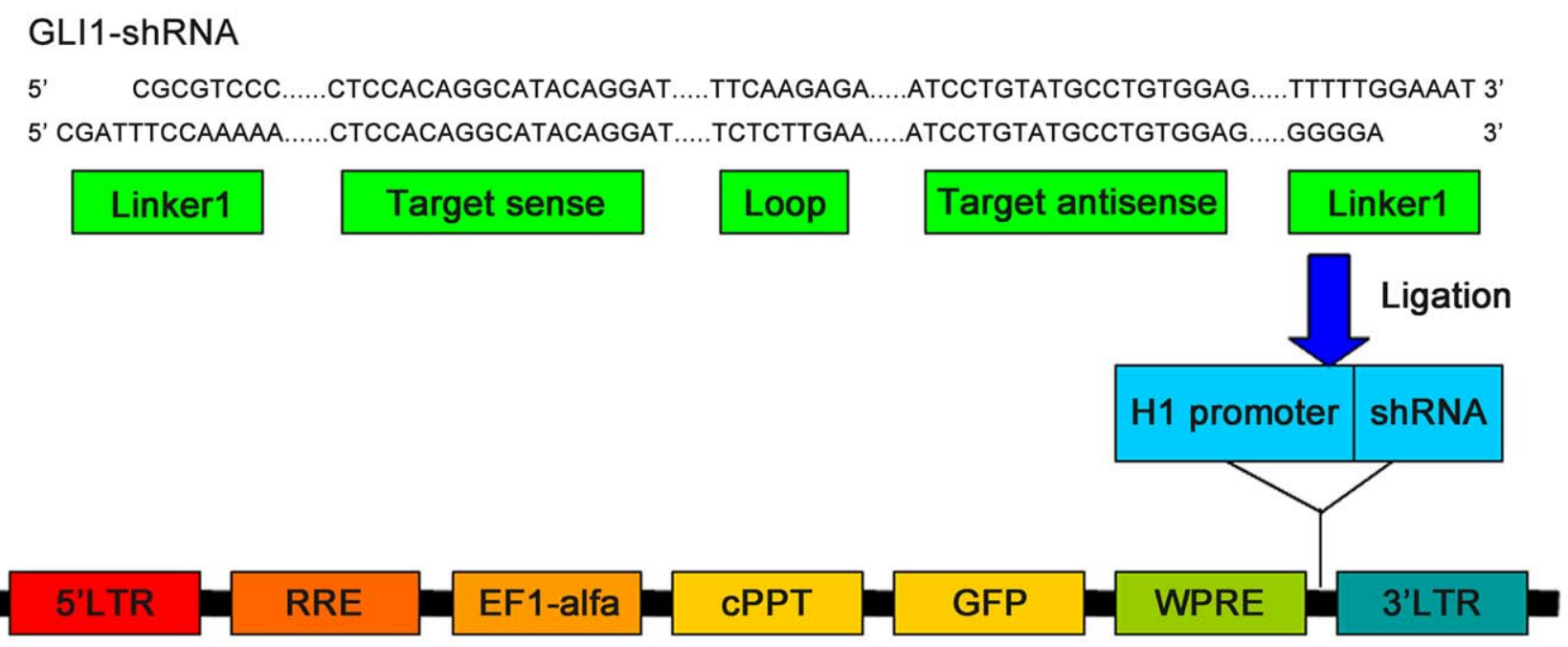

Figure 1. Construction of the pLVTHM vector encoding anti-GLI1 shRNA. For the GLI1-shRNA vector, two single strand DNAs encoding two linkers, the target sequences and a loop element, were synthesized. These were annealed to double stranded DNA, and ligated into the pLVTHM following Mlul and Call digestion. The short hairpin form of shGLI1 is expressed under the control of human $\mathrm{H} 1$ promoter. The vector also contains a human EF1- $\alpha$ promoter driving the GFP marker gene for tracking transduced cells. The vectors were generated by transient transfection of pRsv-REV, pMDlg-pRRE, pMD2G, and the shRNA encoding pLVTHM into 293T cells. Abbreviations: RRE, Rev response element; cPPT, central polypurine tract; EF1- $\alpha$, human elongation factor $1-\alpha$ promoter; $H 1$, the human $\mathrm{H} 1$ promoter; GFP, green fluorescent protein; PRE, human hepatitis virus posttranscriptional regulatory element.

doi:10.1371/journal.pone.0018434.g001

PCR using the forward primer, 5'-GAGGATCGCGGGGTACGGGTCGCGACGATGTTCAACTCGATGACGCCAC-3'; and reverse primer, 5'-TCATCGTTGTAGTCGCTAGCGGCACTAGAGTTGAGGA-3'; then inserted into a pGC-FUEGFP-3FLAG Vector (GeneChem Company, Shanghai, China). Transformants were analyzed by sequencing. The resultant 3320bp fragment was confirmed by sequencing (Figure S1) and compared with the sequence of the GLIl gene expression region in GenBank (NM_005269.2). To produce lentiviral stock, 293FT cells were cultured to $70-85 \%$ confluence the following day. The complete culture medium was removed. Cells were then exposed to $5 \mathrm{~mL}$ medium (Opti-MEM; Invitrogen) with complexes containing packaging helper construct (GeneChem Company, Shanghai, China), $20 \mu \mathrm{g}$ expression plasmid DNA (pGC-FUEGFP-3FLAG-GLI1), or control plasmid DNA (pGG-FU-EGFP3FLAG) with $100 \mu$ lipofectamine 2000 (Invitrogen, USA) in the presence of polybrene $(8 \mu \mathrm{g} / \mathrm{mL}$, Sigma-Aldrich, St. Louis, MO, USA). After incubation for 24 hours, the infection medium was replaced with complete culture medium. Lentivirus-containing supernatants were harvested 72 hours after transfection. The supernatants were centrifuged to remove pellet debris and stored at $-80^{\circ} \mathrm{C}$. Titers of $2-5 \times 10^{7} \mathrm{TU} / \mathrm{ml}$ were routinely achieved.

\section{Lentiviral Transfection}

Cells $\left(1 \times 10^{5}\right)$ in a six-well plate were transfected with the lentiviral vector at a multiplicity of infection $(\mathrm{MOI})=5(\mathrm{PANC}-1)$ or 20 (BxPC-3) in the presence of $8 \mu \mathrm{g} / \mathrm{ml}$ polybrene (SigmaAldrich, St. Louis, MO, USA). After 72 hours of transfection, the medium was replaced with $2 \mathrm{ml}$ complete culture medium. 48 hours after transfection, GLIl expression was established by real time-PCR and Western blot analysis.

\section{Flow Cytometry}

Cells were adjusted to $1 \times 10^{6}$ cells $/ 100 \mu \mathrm{L}$ and used for flow cytometry. A total of 10,000 events were analyzed to determine transfection efficiency using FACS Calibur (Becton Dickinson, USA) Cell-Quest software.

\section{qRT-PCR}

Real-time quantitative reverse-transcription polymerase chain reaction (qRT-PCR) analysis was performed with the ABI Prism 7900HT Sequence Detection System (Applied Biosystems, CA, USA). GLIl and RegIV mRNA expression was analyzed by qRTPCR using SYBR Green Dye. $\beta$-actin was used as the housekeeping gene. Target gene expression was normalized to $\beta$-actin and analyzed by the $2^{-\triangle \Delta C T}$ formula. The primer sequences are as follows: GLIl, forward: TTCCTACGAGAGTCGCAAGT, reverse: CCCTATGTGAAGCCGTATTT, RegIV, forward: GGCTGAGATGAACGCAAAG, reverse: TGAGAGGGAAGTGGGAAGAG. $\beta$-actin, forward: AAGGGACTTCGTGTAACAATGCA, reverse: CTGGAACGGTGAAGGTGACA. All reactions were performed at least three times.

\section{Western blot analysis}

Cells were rinsed twice in PBS, then lysed for 2 hours in RIPA lysis buffer on ice and centrifuged at 12,000 rpm for 10 minutes at $4^{\circ} \mathrm{C}$. Protein concentration was determined by the standard BCA method $\left(\mathrm{BCA}^{\mathrm{TM}}\right.$ Protein Assay Kit, Pierce, USA). $50 \mu \mathrm{g}$ of total protein was separated by SDS-PAGE using $6 \%$ or $12 \%$ polyacrylamide gel with Mini-PROTEAN Tetra Cell (Bio-Rad, USA). GLIl and RegIV protein in gel was transferred to a 0.45$\mu \mathrm{m}$ nitrocellulose membrane with Mini Transfer Cell and Transblot SD Semi-Dry Transfer Cell (Bio-Rad, USA) respectively.

The immunoreagents used for Western blot were rabbit monoclonal antibody against GLI1 (1:200; Santa Cruz, USA) and goat polyclonal anti-RegIV antibody (1:100; Santa Cruz, USA). Mouse polyclonal anti- $\beta$-actin antibody (1:5000; Santa Cruz, USA) was used as loading control. The blots were developed by a standard enhanced chemiluminescence (ECL) method (Pierce, USA). All experiments were repeated several times and gave similar results.

\section{Immunohistochemistry}

Tumor sections were deparaffinized, rehydrated, and treated with $10 \mathrm{mM}$ citrate buffer $\left(\mathrm{pH} \mathrm{6.0)}\right.$ at $95^{\circ} \mathrm{C}$ to retrieve antigens. 
After quenching endogenous peroxidase activity with $\mathrm{H}_{2} \mathrm{O}_{2}$ and blocking with $10 \%$ normal horse serum, the sections were incubated sequentially with the primary antibodies goat antiRegIV (1:100; Santa Cruz, California, USA), rabbit anti-GLI1 (1:200; Santa Cruz, California, USA), biotinylated secondary antibodies, and the ABC reagent (Gene Tech, Shanghai, China). The immunostaining was visualized with 3.3-diaminobenzidine (Gene Tech, Shanghai, China). The sections were then counterstained with hematoxylin. Negative controls were performed in each case by replacing the primary antibody with PBS.

\section{Chromatin immunoprecipitation (CHIP)}

We modified the previously reported protocol [31] for chromatin immunoprecipitation (CHIP). In brief, PANC-1 cells $\left(3 \times 10^{7}\right)$ were cross-linked with $1 \%$ formaldehyde. The fixation reaction was stopped by adding $10 \mathrm{ml}$ Glycin $(0.125 \mathrm{M})$, then chromatin was collected with $1 \mathrm{~mL}$ IP buffer containing protease inhibitor cocktails. Chromatin was sheared by using a sonicator with a $4 \mathrm{~mm}$ tip probe 3 times for 10 second pulses $(60 \mathrm{~W}, 80 \mathrm{~W}$, and $100 \mathrm{~W}$, respectively, $90 \mathrm{~s}$ intervals) in an ice box. Crosslinking was reversed by adding $20 \mu \mathrm{L}$ of $5 \mathrm{M} \mathrm{NaCl}$ overnight at $65^{\circ} \mathrm{C}$. DNA was extracted using phenol/chloroform assay. $20 \mu \mathrm{L}$ of DNA was electrophoresed on a $1.5 \%$ agarose gel and the rest was preserved at $-20^{\circ} \mathrm{C}$ as INPUT DNA. Soluble chromatin was immunoprecipitated with $2 \mu \mathrm{g}$ anti-GLIl rabbit monoclonal antibody (Santa Cruz, California, USA). The $2 \mu \mathrm{g}$ mouse IgG (Santa Cruz, California, USA) was added as a random control, RNA polymerase II as a positive control, and $\beta$-actin antibody as a negative control. DNA-protein immune complexes were eluted and reverse cross-linked, and DNA was extracted with phenol/ chloroform and precipitated. The presence of the RegIV promoter domain containing GLIl motifs in immunoprecipitated DNA was identified by PCR using the following primers: RegIV-A for site 1 (118 bp), forward: 5'-5-TGGTCGCTTCGAGACTTA-3-3', reverse: 5' - TCGAGTATAGATGGCAAA -3'. RegIV-B for site 2 (131 bp), forward: 5'-CTAACGCTTTGCCATCTA -3', reverse: 5'-GACGTGGACACTGAACCTTG-3'. RegIV-C for site 3 (70 bp), forward: 5'-CTATGGTGCTCACAAGGA-3', reverse: 5'-GTGTTACATAACGGGTTT-3'. RegIV-D for site4 (70 bp), forward: 5'-TGTAACACACTCTGTTGATGTAAGG-3', reverse: 5' - CTATTTGAGCTTCTCGCGCAG-3'. RegIV-E for sites 3 and 4 (226 bp), forward: 5'-CTCGGAAGGTTTCTAATC-3', reverse: 5'- TTCAACATGGGTGAGTTT-3'. RegIV-F for sites 3 and 4 (481 bp), forward: 5'-CTATGCTGCTCACAAGGA-3', reverse: 5'-AGACGGCTTCAGAATGTA-3'. RegIV-G for site 5 (315 bp), forward: 5'-TTCCTGAGGCAAGAAGAT-3', reverse: 5'-CGAAGATTTAACACAACA-3'. The PCR conditions for the RegIV promoter region were: denaturation 30 seconds at $94^{\circ} \mathrm{C}$, annealing $30 \mathrm{~s}$, elongation 1 minute at $72^{\circ} \mathrm{C}$. Annealing temperatures for RegIV-A-G were $55^{\circ} \mathrm{C}, 56^{\circ} \mathrm{C}$, $56^{\circ} \mathrm{C}, 47^{\circ} \mathrm{C}, 56^{\circ} \mathrm{C}, 56^{\circ} \mathrm{C}$, and $52^{\circ} \mathrm{C}$, respectively. The amplification of the RegIV promoter region was analyzed after 35 cycles. All experiments were repeated at least three times.

\section{Electrophoretic Mobility Shift Assays (EMSA)}

Nuclear extracts were prepared with NE-PER Nuclear and Cytoplasmic Extraction Reagents (Pierce, Rockford, USA). EMSA and supershift EMSA with digoxin-labeled probes were performed using the DIG-Gel shift kit according to the manufacturer's instructions (Roche, Basel, Switzerland). The sequences of the oligonucleotides used were 5'-AGAACATGGATGATCATGTCA-3' (binding motif underlined). Mutant oligonucleotides used were 5'-AGAACAAAAAATTTTATGTCA-3'. In the supershift study, $5 \mu \mathrm{g}$ rabbit monoclonal antibody against GLIl was incubated with $5 \mu \mathrm{g}$ of nuclear extract on ice for 30 minutes before addition of the labeled probe, and further incubated on ice for 30 minutes. The entire $20 \mu \mathrm{l}$ binding reaction was resolved on a $7 \%$ polyacrylamide gel and transferred to a positively charged nylon membrane (Bio-Rad, USA) in $0.5 \times$ Tris borate-EDTA buffer.

\section{Statistical analysis}

Quantitative data are expressed as the mean \pm standard deviation (SD). Real-time PCR data was analyzed according to the differences of target gene expression by the paired t-test and were $2^{-\triangle \Delta C T}$ transformed before analysis. The relationship between GLI1 and RegIV expression was analyzed using Spearman. IHC data was analyzed using the Chi-squared test. A p-value of less than 0.05 was considered statistically significant.

\section{Results}

GLI1 and RegIV expression in pancreatic cancer tissues

To study GLIl and RegIV expression in PG, qRT-PCR and IHC were used in 12 paired biopsy tissues. GLIl expression was higher in 9 cases (9/12) compared with adjacent normal pancreatic tissues $(p=0.011$; Figure 2$)$; RegIV expression was higher in 9 cases $(9 / 12)(\mathrm{p}=0.011$; Figure 2). There was a positive correlation between GLIl and RegIV in PG tissues $(\mathrm{R}=0.795$, $\mathrm{p}<0.0001$; Figure 2). On IHC, we found RegIV to be expressed only in beta cells of normal endocrine pancreatic tissues, which confirmed Oue's report [37]. On IHC, GLIl and RegIV expression were higher in most $\mathrm{PC}$ compared with normal tissues $(15 / 21$ versus $4 / 21, p=0.001 ; 14 / 21$ versus $5 / 21, p=0.005$; respectively; Figure 3). 15 of $21 \mathrm{PC}$ cases had high expression of GLIl protein, among which 11 cases expressed high levels of RegIV protein $(p=0.001$; Figure 3$)$.

\section{The correlation between GLI1 and RegIV}

We tested GLI 1 and RegIV expression in 5 PC cell lines by qPCR and Western blot. There was a positive correlation between the level of GLIl and RegIV mRNA and protein $(\mathrm{R}=0.958$, $\mathrm{p}=0.011$ and $\mathrm{R}=0.939, \mathrm{p}=0.018$, respectively; Figure 4). GLI1 and RegIV were overexpressed in PC versus normal pancreatic cells.

\section{RegIV expression changed with GLI1 expression in PANC- 1 and BxPC-3}

To further verify the relationship between GLI1 and RegIV in PG cells, we designed and constructed shRNA-GLIl lentiviral vector, and transfected it into PANG-1, a PG cell line with the highest expression of GLIl (Figure 4). 48 hours after transfection, efficiency of transfection was shown by flow cytometry (FCM) to be more than 95\% (Figure S2); stable fluorescence could still be detected even after 20 passages (Figure S3).

Afterwards, qRT-PGR and Western blot were used to detect RegIV expression in GLI1-shRNA-PANC-1 cells. Cells without transfection were used as controls, while cells transfected with scramble shRNA were used as negative controls. RegIV mRNA decreased by $94.7 \pm 0.3 \%$ when GLIl mRNA decreased by $82.1 \pm 3.2 \%$. RegIV protein decreased by $84.1 \pm 0.5 \%$ when GLI protein decreased by $76.7 \pm 2.2 \%$ (Figure 5 ). This suggested that RegIV expression decreased when GLIl was silenced by RNAi.

We further designed and constructed a lentivirus vector that expressed GLI1, and transfected it into BxPC-3, with the lowest GLI1 expression in the 5 cell lines (Figure 4), to determine whether RegIV expression changed along with GLI1. 48 hours after transfection, qRT-PCR and Western blot were used to detect RegIV in the LV-GLI1-BxPC-3 cells. Cells without transfection were used as controls, while cells transfected with empty lentivirus 
A

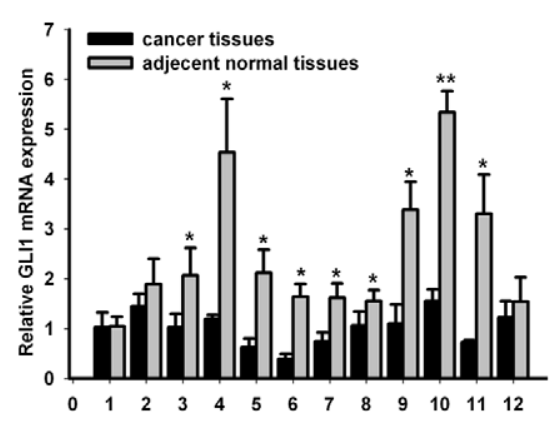

B

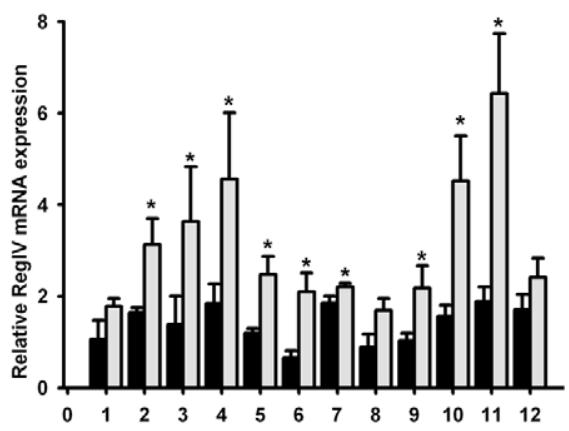

C

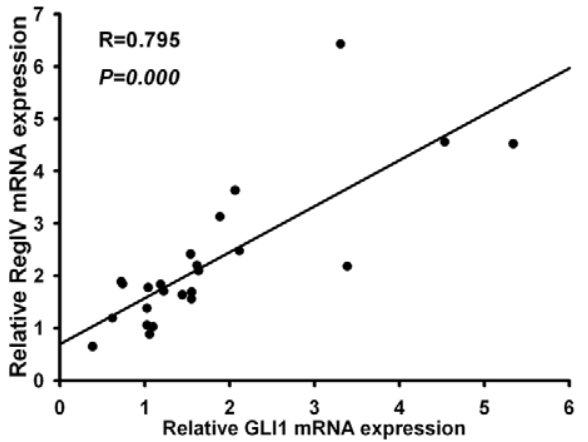

Figure 2. GLI1 and RegIV mRNA expression in PC tissues and adjacent normal tissues. The expression of GLI1 RegIV mRNA in 12 pairs of PC tissues and adjacent normal tissues(A-B). DNA from the samples were collected from surgical biopsies, and relative GLI1 and RegIV mRNA expression were detected by qRT-PCR. Statistical correlation between the expression of GLI1 and RegIV mRNA in 12 pairs of PC tissues and adjacent normal tissues was analyzed by Spearman's test (C). All results were normalized to $\beta$-actin mRNA expression. The data are presented as the mean \pm SD and were calculated by the paired t-test. Significantly different between two groups: ${ }^{*} p<0.05 .{ }^{* *} p<0.01$. NS: not significant. doi:10.1371/journal.pone.0018434.g002

vector were used as negative controls. RegIV mRNA increased by $729.1 \pm 4.3 \%$ when GLIl mRNA increased by $924.5 \pm 5.3 \%$. RegIV protein increased by $339.0 \pm 3.7 \%$ when GLIl protein increased by $362.1 \pm 3.5 \%$ (Figure 5). This implies that RegIV expression increased when GLIl was overexpressed. Based on these results, we concluded that GLI1, a transcription factor, might regulate RegIV gene expression.

\section{Identification of candidate Gli1 binding sites in the RegIV} promoter

The positive correlation between GLIl and RegIV in both PC tissue and cell lines prompted us to search the RegIV promoter for potential GLI1 binding sites to the DNA consensus sequence 5'GACCACCCA-3' [42]. Database analysis revealed four potential sites located upstream of the transcriptional start site (Figure 6). The homology of each GLIl binding site to the canonical consensus sequence varied from $67 \%$ (sites 1, 2, 3, and 5) to 78\% (site 4), which suggested that the RegIV gene promoter might bind to GLI1.

\section{Confirmation of GLI1 protein bound to promoter region} of RegIV gene by CHIP

The sonicated chromatin solution assay showed that the total DNA fragment appeared smeared in the $100 \mathrm{bp}$ to $1 \mathrm{~kb}$ range in the $80 \mathrm{~W}$ group (Figure S4). The result of DNA electrophoresis
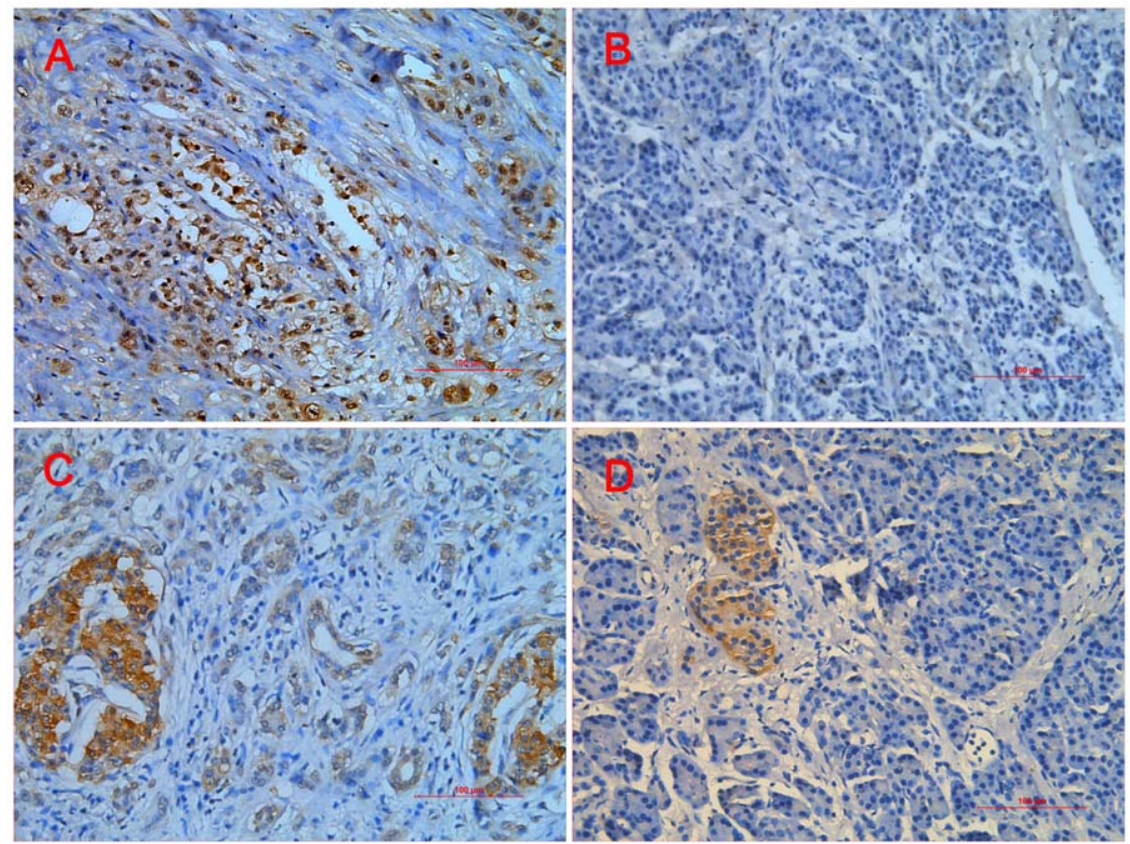

Figure 3. Expression of GLI1 and RegIV proteins was analyzed by IHC in PC and adjacent normal tissues. All samples were collected, formalin-fixed, paraffin-embedded, and detected by IHC. Representative pictures are shown. Positive staining of GLI1 was observed at the PDAC cell nucleus (A); however, adjacent normal tissues exhibited no or faint staining for GLI1 (B). In adjacent normal pancreatic tissues, only islet cells showed positive staining of RegIV (D), while the positive staining of RegIV was observed as well as goblet-like cytoplasm granules in PC tissues (C). All photomicrographs were obtained at $\times 200$ magnification. Scale bars, $100 \mu \mathrm{m}$.

doi:10.1371/journal.pone.0018434.g003 

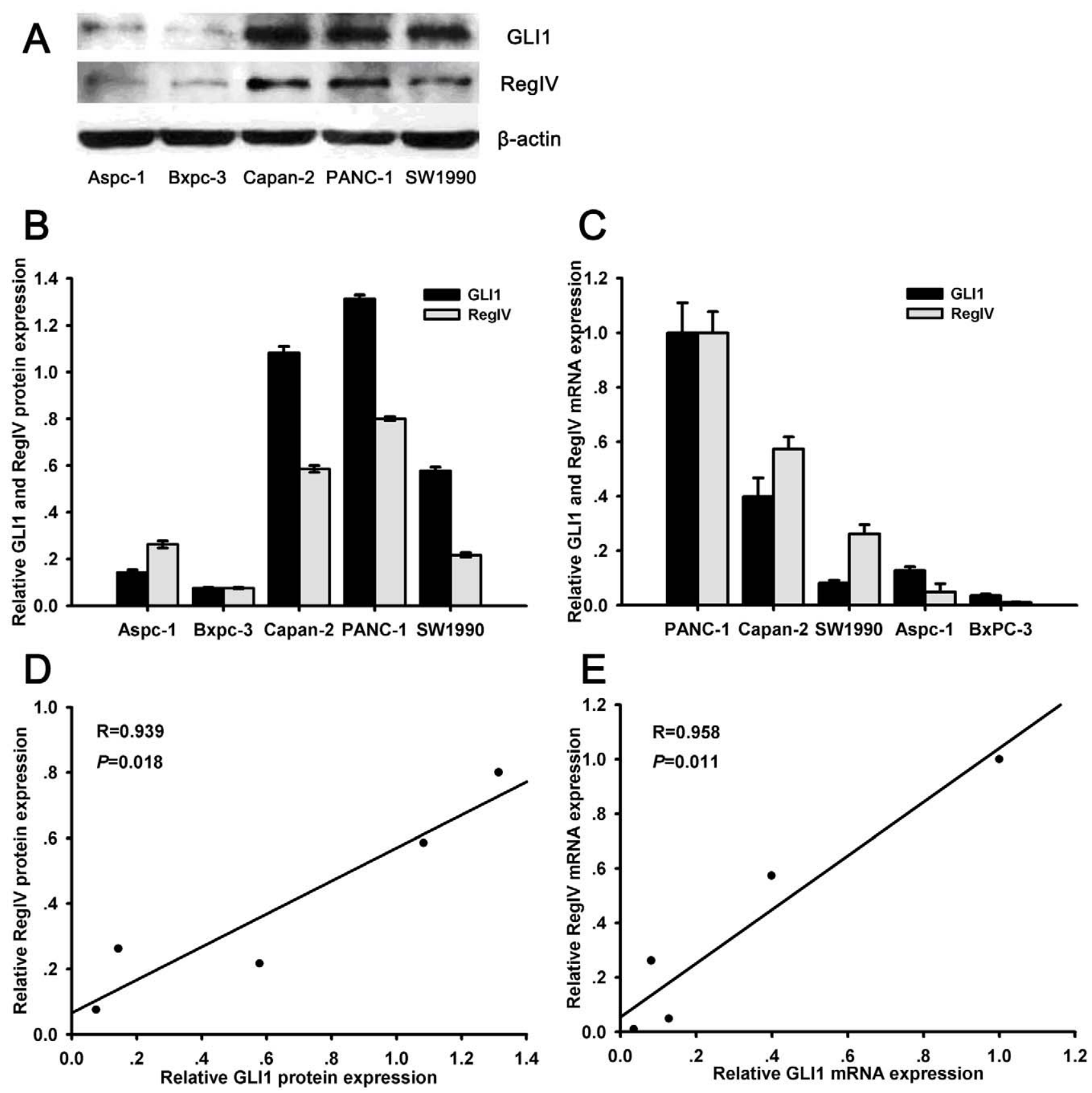

Figure 4. The expression of GLI1 and RegIV in 5 PC cell lines. Expression of GLI1 and RegIV proteins in 5 pancreatic cancer cell lines as detected by Western blot analysis on cell extracts, using anti-GLI1 and anti-RegIV antibodies (A). $\beta$-actin was used as the loading control in all experiments. The results were quantified by determining the intensities of the bands compared with that of $\beta$-actin (B). Statistical correlation between expression of GLI1 and RegIV protein in 5 PC cell lines was analyzed by Pearson's test (C). Relative GLI1 and RegIV mRNA expression were examined by qRT-PCR (D). The expression of GLI1 and RegIV mRNA was normalized to $\beta$-actin mRNA expression. Statistical correlation between the expression of GLI1 and RegIV mRNA in 5 PC cell lines was analyzed by Pearson's test (E). All data are presented as the mean \pm SD of three independent experiments.

doi:10.1371/journal.pone.0018434.g004

showed the predicted DNA band in INPUT, GLIl-Ab, and postive control groups using human RegIV primer-D-F, and not in the IgG and negative control groups (Figure 7). Only INPUT and the positive control showed the predicted band using human RegIV primer-A-C, G but not in GLI-Ab, IgG, and negative groups (data not shown). The results of sequence analysis showed that the sequences were the same as that of the RegIV gene promoter of site 4 (Figure S5, S6, S7). All data suggested that
GLIl was bound to the RegIV gene promoter of site 4 (GATCATCGA), and regulated RegIV in PC through the $\mathrm{HH}$ signaling pathway.

Confirmation of GLI1 bound to the RegIV promoter by EMSA

As described above, the GLIl binding site in the promoter region of the RegIV gene was confirmed with ChIP-PCR. We 
A
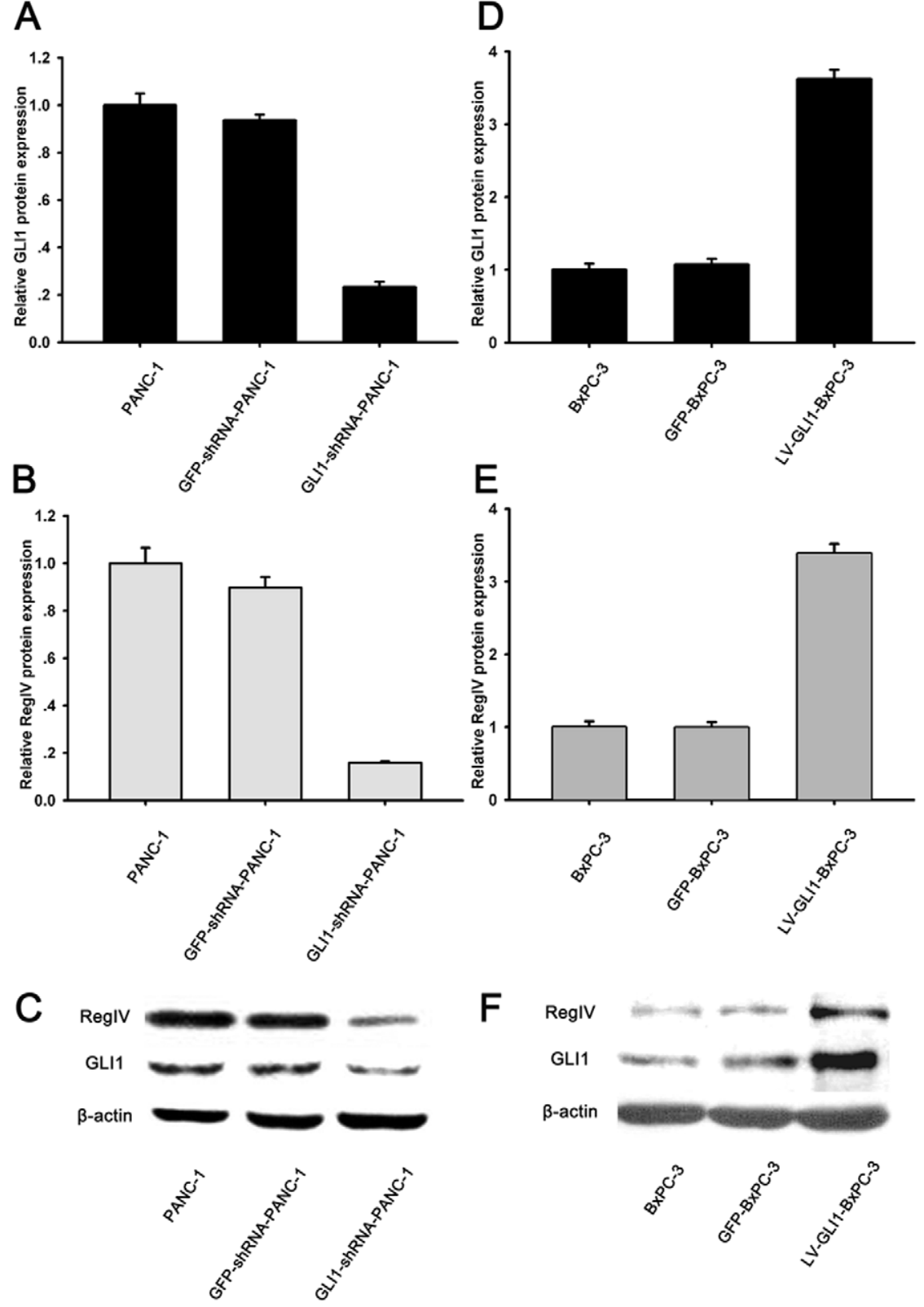

Figure 5. RegIV expression changed with GLI1 in PC cells. PANC-1 cells were transfected with GLI1-shRNA or GFP-shRNA, BxPC-3 cells were transfected with LV-GLI1-eGFP or LV-eGFP, then expression of GLI1 mRNA relative to that of $\beta$-actin mRNA was assessed by qRT-PCR (A, D). After transfection, expression of GLI1 proteins was analyzed by Western blot $(C, F)$. The inset shows a substantial decrease in RegIV expression by real-time RT-PCT (B, E) and Western blot analysis (C, F). The results were normalized to that of $\beta$-actin expression. All data are presented as the mean \pm SD of three independent experiments.

doi:10.1371/journal.pone.0018434.g005

then used EMSA assays to directly address whether GLI1 binds RegIV in vivo. We synthesized specific oligonucleotides containing the GLIl element present in the RegIV promoter in EMSA experiments with nuclear extracts from PANC-1 cell lines. As shown in Figure 8, incubation of PANG-1 cells extracts with the biotin-labeled GLIl-RegIV sequence produced a DNA-protein band shift. These DNA-protein complexes were specific to the GLI1 site by successful competition assays using different folds of excess unlabeled GLI1-RegIV and mutant labeled GLI1-RegIV oligonucleotides. To confirm the binding of GLI1 to the GLI1RegIV sequence, these EMSA reactions were further incubated with anti-GLI1 antibody. As shown in Figure 8, the addition of this antibody resulted in a supershifted complex in addition to the
DNA-protein band. These data demonstrated the presence of GLIl in the nuclear protein complex that binds the GLI1 binding site of the RegIV promoter (-528 -520).

\section{Discussion}

In this study, we confirmed that GLIl and RegIV were overexpressed in PC tissue and cell lines, confirmed by other reports [12,20,32]. We also demonstrated a significantly positive correlation between the expression of GLIl and RegIV. RNA interference and overexpression experiments showed that RegIV expression changed with GLIl expression in PC cell lines; this was confirmed by CHIP and EMSA. This is the first report 


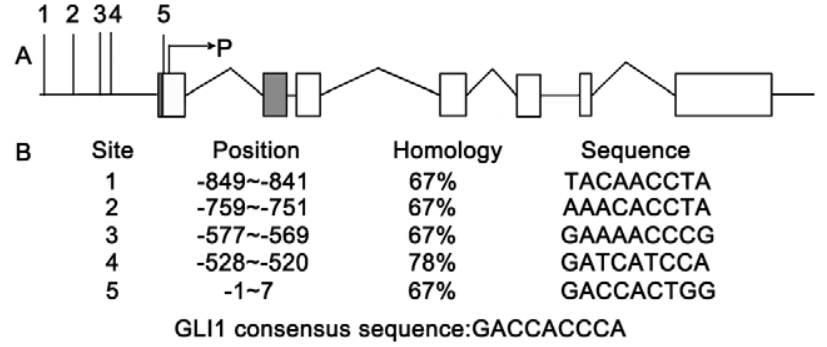

Figure 6. Potential GLI1 binding sites on the RegIV promoter and homology to the GLI1 consensus sequence. (A) Location of the potential GLI1 binding sites (numbers $1-5$ ) in relation to the structure of the gene. $P$ represents the transcriptional start site. The gray frame represents the variable splicing site. Blank frames represent exons. (B) Position of the binding sites on the promoter in relation to the $P$ transcriptional start site and the sequence homology to the GLI1 consensus binding sequence, GACCACCCA. doi:10.1371/journal.pone.0018434.g006

that GLI1 can modulate RegIV expression by binding to the RegIV gene promoter, and that GLI1 is a RegIV transcriptional factor.

The $\mathrm{HH}$ signaling pathway, including transcription factor GLI1, is involved in the development of many kinds of cancers, including PC [12,13,32-35]; however, the mechanism has not been fully elucidated. Thus far, only a few downstream targets of GLIl have been identified, including GLIl, PTCH, HHIP, CGND, Snail, Bcl-2, cyclin D2, FOX-F1, -L1, -M1, Follistatin, and N-Myc [36]. We demonstrated that the HH-GLI1 signaling pathway could regulate RegIV expression by a serie of experiments, including CHIP and EMSA. In our literature review, we learned that RegIV expression in different cell types was associated with regeneration, and cell growth, survival, adhesion, and resistance to apoptosis. RegIV is systematically overexpressed in stomach [24], colon [25,26], and pancreas cancers $[27,28]$ and in diseases that predispose to colon cancer such as ulcerative colitis [29]. IHC analysis has confirmed RegIV expression in gastric, colorectal, and pancreatic carcinoma $[27,37,38]$, and that RegIV has a potential role in diagnosing digestive tract neuroendocrine tumors [39]. RegIV gene amplification is an early event in pancreatic cancer development [30], and elevated RegIV was found in the sera of patients with PC [28]. PC-derived cells overexpressing RegIV protein grew more rapidly and were more resistant to gemcitabine treatment [30]. RegIV overexpression was thought to be associated with an unfavorable response to adjuvant chemoradiotherapy in patients with PC [40]. Other studies showed that RegIV was associated with a relatively favorable prognosis in patients with gallbladder carcinoma after surgical resection [41]. Thus, we concluded that the HH/GLI1/RegIV cascade may be an important pathway in PG development.

Chromatin immunoprecipitation (CHIP) is a reliable procedure used to determine whether a protein binds to or is localized to a specific DNA sequence in vivo. Through CHIP and promoter analysis, we identified a direct transcriptional target gene of GLI1, although the GLI1-binding element (GATCATCCA) showed a 2 nucleotide difference (underlined) from a previously identified 9nucleotide GLI1-binding sequence (GACGACGCGA) [42]. EMSA is one of the most sensitive methods for studyting protein-DNA interactions. This procedure can determine if a protein or mixture of proteins is capable of binding to a given DNA sequence. "Supershift assay" is a term used to unambiguously identify a protein present in the protein-nucleic acid complex. The EMSA and supershift assay also confirmed GLIl to be bound on site 4 of the RegIV promoter motif. Those results suggested that GLIl can bind to RegIV gene promoters on site 4 in vivo. Based on these results, we concluded that GLIl transcriptionally regulates RegIV gene in PC cells.

Although the biological function of RegIV is poorly understood, it has been reported that RegIV may function as a growth and antiapoptotic factor in gastric, colon, and pancreatic cancers $[27,29,43,44]$. The expression of RegIV may contribute to liver metastasis through induction of MMP7 by RegIV [44], and is a

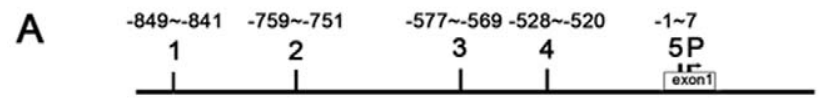

primer-A

primer-B $\quad-843 \quad-713$

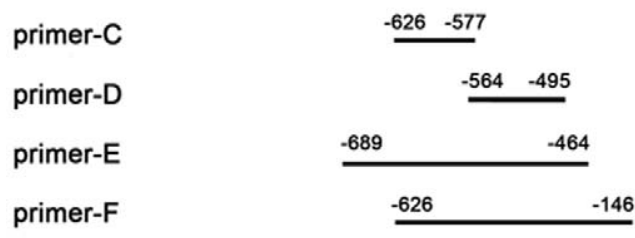

primer-G
$-45$ 270

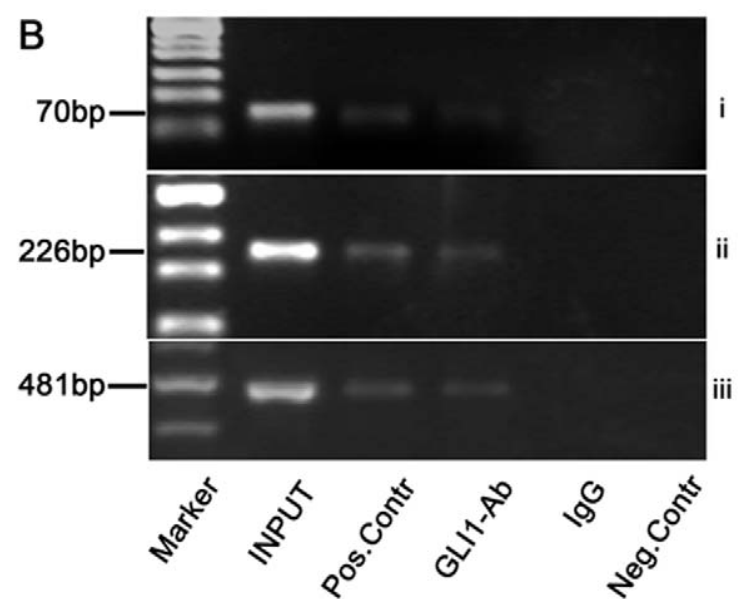

Figure 7. Modulation of GLI1 binding on RegIV promoter was assessed by Chromatin immunoprecipitation (ChIP) assay. The locations of RegIV primer-A-G in the promoter region of RegIV gene. The numbers on the schematic of the RegIV gene (numbers 1-5) correspond to the potential GLI1 binding sites. P represents the transcriptional start site. Lysates from PANC-1 cells were subjected to Chromatin immunoprecipitation by anti-GLI1 antibody. Human RegIV primer-A-G were used to amplify the RegIV promoter region containing the putative GLI1-binding site. Sonicated chromatin were used as INPUT DNA control. IgG, RNA polymerase II, and $\beta$-actin Ab were used as random controls, positive controls, and negative controls. (B) Only INPUT, positive control, and GLI1-Ab showed the predicted band in ethidium bromide-stained agarose gels using the CHIP-PCR products which were amplified by RegIV primer-D(i), RegIV primer-E(ii), and RegIV primer-F(iii). No detectable transcript was observed in amplified template from IgG or negative control and a positive control lane confirmed the expected fragment. Molecular weight standards (Marker) were used to estimate the size of the amplified bands.

doi:10.1371/journal.pone.0018434.g007 


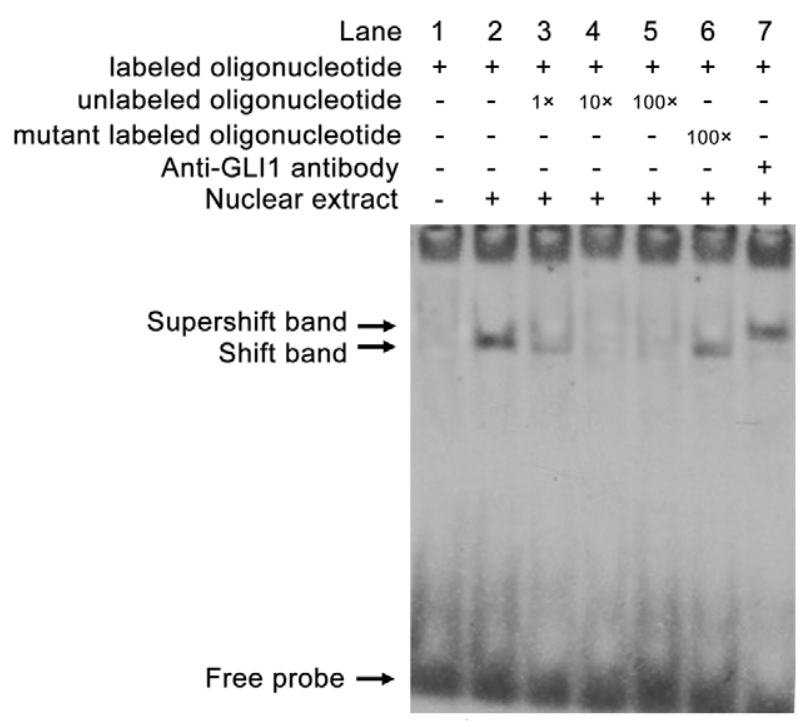

Figure 8. Analyses of the binding of GLI1 to the Reg IV promoter by Electrophoretic Mobility Shift Assays (EMSA). EMSA was performed with nuclear extracts of PANC-1 cells (lanes 2 to 7 ) or without nuclear extracts (lane 1). The RegIV probe was generated by annealing single-stranded and end-labeled oligonucleotides containing the RegIV promoter region (nucleotides -528 -520). Competition experiments were performed using 1-fold (lanes 3), 10-fold (lanes 4), and 100-fold (lanes 5) excess of unlabeled oligonucleotides, respectively (lanes 3 to 5) or 100-fold mutant labeled oligonucleotides (lane 6). For super-shift, anti-GLI1 antibody (lane 7) was incubated with nuclear extracts before being added to the reaction.

doi:10.1371/journal.pone.0018434.g008

potent activator of the EGFR/Akt/AP-1 signaling pathway in colon cancer cells. It also increases the expression of Bcl-2, Bcl-xl, and survival proteins, all associated with the inhibition of apoptosis [44]. However, the role of RegIV in migration and invasion, and whether GLIl contributes to proliferation, migration, and invasiveness through RegIV regulation in PG is still unclear. Whether RegIV is transcriptionally regulated by GLI1, thus imposing its effect on pancreatic carcinogenesis, the pathways responsible require further investigation. The coherence of different molecular events would be partly elucidated by revealing the mechanism of transcriptional regulation between GLIl and RegIV, which may be determined by investigating the effects of GLIl and RegIV on common signaling pathways such as the EGFR/Akt/AP1 cascade, as reported recently both in $\mathrm{HH}$ and RegIV. Our work may contribute to the body of research on pancreas carcinogenesis and provide insight into the correlated network of signaling pathways through the GLI1/RegIV axis.

In conclusion, the SHH-GLIl signaling pathway regulates the transcription of RegIV gene in PG. This is the first report that demontrates GLII as a transcriptional factor that regulates RegIV expression in PC. Our work may help to elucidate the molecular mechanism of the SHH-GLIl signaling pathway and promote earlier diagnosis and treatment of PC. The newly identified GLI1/ RegIV axis provides a new insight into PC pathogenesis. Additional studies are required to determine whether the biological behavior of GLIl in PC may be achieved by regulating RegIV.

\section{References}

1. Bardeesy N, DePinho RA (2002) Pancreatic cancer biology and genetics. Nat Rev Cancer 2: 897-909.

\section{Supporting Information}

Figure S1 The result of sequence analysis of positive clone products in overexpression-GLI1 lentiviral vector construction. The resultant 3320 -bp fragment was confirmed by sequencing which is same with the sequence of the GLIl gene expression region in GenBank (NM_005269.2).

(TIF)

Figure S2 Transduction efficiency of PANC-1 cells by lentivirus vector were evaluated by FGM. Cells were transfected with GFP-vector. Transduction efficiency based on the fluorescent signalwas analyzed by FCM. (A) PANC-1 cells without transfection were used as the blank control; (B) Cells transfected with GFP-shRNA as random control; (C) Cells transfected with GLI1-shRNA as experiment group.

(TIF)

Figure S3 Transduction efficiency of PANG-1 cells by lentivirus vector, phase contrast and GFP expression under a fluorescent microscope. Transduction efficiency of PANC-1 cells by GLI1 silencing vector. PANC-1 cells were transfeced with the GLI1-shRNA vector. The corresponding phase-contrast image(left panel), the GFP fluorescence (middle panel) and the merged image (right panel) are shown at a magnification of $\times 200$. GFP expression reveals high transduction efficiency, with more than $90 \%$ of cells being transduced.

(TIF)

Figure S4 Electropheretogram of sonicated chromatin solution in different conditions. Sonicated chromatin solution in different conditions were electrophoresed on $1.5 \%$ agarose gel containing ethidium bromied. DNA sizes appear smear at a range of $100 \mathrm{bp}$ to $1 \mathrm{~kb}$ range in $80 \mathrm{~W}$ group.

(TIF)

Figure S5 The result of sequence analysis of CHIP products which amplified by RegIV primer-D. The result showed that the sequence amplified with RegIV primer-D is the same as that of RegIV gene promoter region containing GLI1binding site 4 .

(TIF)

Figure S6 The result of sequence analysis of CHIP products which amplified by RegIV primer-E. The result showed that the sequence amplified with RegIV primer-E is the same as that of RegIV gene promoter region containing GLI1binding site 4 .

(TIF)

Figure 57 The result of sequence analysis of CHIP products which amplified by RegIV primer-F. The result showed that the sequence amplified with RegIV primer-F is the same as that of RegIV gene promoter region containing GLI1binding site 4 .

(TIF)

\section{Author Contributions}

Conceived and designed the experiments: FW XPW. Performed the experiments: FW LX GYH XFX WHM LJY YSH. Analyzed the data: FW LX. Contributed reagents/materials/analysis tools: XPW CYG. Wrote the paper: FW AWK SSH. 
3. Herszényi L, Tulassay Z (2010) Epidemiology of gastrointestinal and liver tumors. Eur Rev Med Pharmacol Sci 14: 249-258.

4. Morris JP, 4th, Wang SC, Hebrok M (2010) KRAS, Hedgehog, Wnt and the twisted developmental biology of pancreatic ductal adenocarcinoma. Nature Reviews Cancer 10: 683-695.

5. Klein WM, Hruban RH, Klein-Szanto AJ, Wilentz RE (2002) Direct correlation between proliferative activity and dysplasia in pancreatic intraepithelial neoplasia (PanIN): additional evidence for a recently proposed model of progression. Mod Pathol 15: 441-447.

6. Kim SK, Hebrok M (2001) Intercellular signals regulating pancreas development and function. Genes Dev 15: 111-127.

7. Prasad NB, Biankin AV, Fukushima N, Maitra A, Dhara S, et al. (2005) Gene expression profiles in pancreatic intraepithelial neoplasia reflect the effects of Hedgehog signaling on pancreatic ductal epithelial cells. Cancer Res 65: $1619-1626$.

8. Nüsslein-Volhard C, Wieschaus E (1980) Mutations affecting segment number and polarity in Drosophila. Nature 287: 795-801.

9. Chiang C, Litingtung Y, Lee E, Young KE, Corden JL, et al. (1996) Cyclopia and defective axial patterning in mice lacking Sonic hedgehog gene function. Nature 383: 407-413.

10. Hebrok M (2003) Hedgehog signaling in pancreas development. Mech Dev 120: $45-57$.

11. Watkins DN, Berman DM, Burkholder SG, Wang B, Beachy PA, et al. (2003) Hedgehog signalling within airway epithelial progenitors and in small-cell lung cancer. Nature 422: 313-317.

12. Berman DM, Karhadkar SS, Maitra A, Montes De Oca R, Gerstenblith MR, et al. (2003) Widespread requirement for Hedgehog ligand stimulation in growth of digestive tract tumours. Nature 425: 846-851.

13. Thayer SP, di Magliano MP, Heiser PW, Nielsen CM, Roberts DJ, et al. (2003) Hedgehog is an early and late mediator of pancreatic cancer tumorigenesis. Nature 425: 851-856.

14. Morton JP, Mongeau ME, Klimstra DS, Morris JP, Lee YC, et al. (2007) Sonic hedgehog acts at multiple stages during pancreatic tumorigenesis. Proc Natl Acad Sci U S A 104: 5103-5108.

15. Ingham PW, McMahon AP (2001) Hedgehog signaling in animal development: paradigms and principles. Genes Dev 15: 3059-3087.

16. Dai P, Akimaru H, Tanaka Y, Maekawa T, Nakafuku M, et al. (1999) Sonic Hedgehog-induced activation of the Glil promoter is mediated by GLI3. J Biol Chem 274: 8143-8152.

17. Lee J, Platt KA, Gensullo P, Ruiz i Altaba A (1997) Glil is a target of Sonic hedgehog that induces ventral neural tube development. Development 124 : 2537-2552.

18. Wang B, Fallon JF, Beachy PA (2000) Hedgehog-regulated processing of Gli3 produces an anterior/posterior repressor gradient in the developing vertebrate limb. Cell 100: 423-434.

19. Aza-Blanc P, Lin HY, Ruiz i Altaba A, Kornberg TB Expression of the vertebrate Gli proteins in Drosophila reveals a distribution of activator and repressor activities. Development 2000;127: 4293-4301.

20. Zhang YW, Ding LS, Lai MD (2003) Reg gene family and human diseases. World J Gastroenterol 9: 2635-2641.

21. Bishnupuri KS, Luo Q, Sainathan SK, Kikuchi K, Sureban SM, et al. (2010) Reg IV regulates normal intestinal and colorectal cancer cell susceptibility to radiation-induced apoptosis. Gastroenterology 138: 616-626.

22. Sekikawa A, Fukui H, Fujii S, Takeda J, Nanakin A, et al. (2005) REG Ialpha protein may function as a trophic and/or anti-apoptotic factor in the development of gastric cancer. Gastroenterology 128: 642-653.

23. Kuniyasu H, Oue N, Sasahira T, Yi L, Moriwaka Y, et al. (2009) Reg IV enhances peritoneal metastasis in gastric carcinomas. Cell Prolif 42: 110-121.

24. Oue N, Hamai Y, Mitani Y, Matsumura S, Oshimo Y, et al. (2004) Gene expression profile of gastric carcinoma: identification of genes and tags potentially involved in invasion, metastasis, and carcinogenesis by serial analysis of gene expression. Cancer Res 64: 2397-2405.
25. Violette S, Festor E, Pandrea-Vasile I, Mitchell V, Adida C, et al. (2003) Reg IV, a new member of the regenerating gene family, is overexpressed in colorectal carcinomas. Int J Cancer 103: 185-193.

26. Zhang Y, Lai M, Lv B, Gu X, Wang H, et al. (2003) Overexpression of Reg IV in colorectal adenoma. Cancer Lett 200: 69-76.

27. Takehara A, Eguchi H, Ohigashi H, Ishikawa O, Kasugai T, et al. (2006) Novel tumor marker REG4 detected in serum of patients with resectable pancreatic cancer and feasibility for antibody therapy targeting REG4. Cancer Sci 97: 1191-1197.

28. Takayama R, Nakagawa H, Sawaki A, Mizuno N, Kawai H, et al. (2010) Serum tumor antigen REG4 as a diagnostic biomarker in pancreatic ductal adenocarcinoma. J Gastroenterol 45: 52-59.

29. Nanakin A, Fukui H, Fujii S, Sekikawa A, Kanda N, et al. (2007) Expression of the REG IV gene in ulcerative colitis. Lab Invest 87: 304-314.

30. Legoffic A, Calvo E, Cano C, Folch-Puy E, Barthet M, et al. (2009) The reg4 gene, amplified in the early stages of pancreatic cancer development, is a promising therapeutic target. PLoS One 4: e7495.

31. Ni Z, Kim ED, Ha M, Lackey E, Liu J, et al. (2009) Altered circadian rhythms regulate growth vigour in hybrids and allopolyploids. Nature 457: 327-331.

32. Nolan-Stevaux O, Lau J, Truitt ML, Chu GC, Hebrok M, et al. (2009) GLI1 is regulated through Smoothened-independent mechanisms in neoplastic pancreatic ducts and mediates PDAC cell survival and transformation. Genes Dev 23: 24-36.

33. Feldmann G, Fendrich V, McGovern K, Bedja D, Bisht S, et al. (2008) An orally bioavailable small-molecule inhibitor of Hedgehog signaling inhibits tumor initiation and metastasis in pancreatic cancer. Mol Cancer Ther 7: 2725-2735.

34. Feldmann G, Dhara S, Fendrich V, Bedja D, Beaty R, et al. (2007) Blockade of hedgehog signaling inhibits pancreatic cancer invasion and metastases: a new paradigm for combination therapy in solid cancers. Cancer Res 67: 2187-2196.

35. Nagai S, Nakamura M, Yanai K, Wada J, Akiyoshi T, et al. (2008) Glil contributes to the invasiveness of pancreatic cancer through matrix metalloproteinase-9 activation. Cancer Sci 99: 1377-1384.

36. Katoh Y, Katoh M (2009) Hedgehog Target Genes: Mechanisms of Carcinogenesis Induced by Aberrant Hedgehog Signaling Activation. Curr Mol Med 9: 873-886.

37. Oue N, Mitani Y, Aung PP, Sakakura C, Takeshima Y, et al. (2005) Expression and localization of Reg IV in human neoplastic and non-neoplastic tissues: Reg IV expression is associated with intestinal and neuroendocrine differentiation in gastric adenocarcinoma. J Pathol 207: 185-198.

38. Li XH, Zheng Y, Zheng HC, Takahashi H, Yang XH, et al. (2010) REG IV overexpression in an early stage of colorectal carcinogenesis: an immunohistochemical study. Histol Histopathol 25: 473-484.

39. Li FY, Ren XB, Xu EP, Huang O, Sheng HO, et al. (2010) RegIV expression showing specificity to gastrointestinal tract and its potential role in diagnosing digestive tract neuroendocrine tumor. J Zhejiang Univ Sci B 11: 258-66.

40. Eguchi H, Ishikawa O, Ohigashi H, Takahashi H, Yano M, et al. (2009) Serum REG4 level is a predictive biomarker for the response to preoperative chemoradiotherapy in patients with pancreatic cancer. Pancreas 38: 791-798.

41. Tamura H, Ohtsuka M, Washiro M, Kimura F, Shimizu H, et al. (2009) Reg IV expression and clinicopathologic features of gallbladder carcinoma. Human Pathology 40: 1686-1692.

42. Kinzler KW, Vogelstein B (1990) The GLI gene encodes a nuclear protein which binds specific sequences in the human genome. Mol Cell Biol 10: $634-642$.

43. Mitani Y, Oue N, Matsumura S, Yoshida K, Noguchi T, et al. (2007) Reg IV is a serum biomarker for gastric cancer patients and predicts response to 5fluorouracil-based chemotherapy. Oncogene 26: 4383-4393.

44. Bishnupuri KS, Luo Q Murmu N, Houchen CW, Anant S, et al. (2006) Reg IV activates the epidermal growth factor receptor/Akt/AP-1 signaling pathway in colon adenocarcinomas. Gastroenterology 130: 137-149. 